\title{
Loss of mitochondrial membrane potential triggers the retrograde response extending yeast replicative lifespan
}

\section{Michael V. Miceli, James C. Jiang, Anurag Tiwari, Jose F. Rodriguez-Quiñones ${ }^{\dagger}$ and S. Michal Jazwinski*}

Tulane Center for Aging and Department of Medicine, Tulane University Health Sciences Center, New Orleans, LA, USA

\section{Edited by:}

Yidong Bai, University of Texas Health

Science Center at San Antonio, USA

Reviewed by:

Lokendra Kumar Sharma, University

of Texas Health Science Center at

San Antonio, USA

Xin Jie Chen, SUNY Upstate Medical

University, USA

\section{*Correspondence.}

S. Michal Jazwinski, Department of

Medicine, Tulane University Health

Sciences Center, 1430 Tulane Avenue,

SL-12, New Orleans, LA 70112, USA.

e-mail: sjazwins@tulane.edu

\section{${ }^{t}$ Present address:}

Jose F. Rodriguez-Quiñones,

Department of Chemistry, College of Sciences, Pontifical Catholic

University of Puerto Rico, Ponce, PR, USA.
In the budding yeast Saccharomyces cerevisiae, loss of mitochondrial DNA (rho ${ }^{0}$ ) can induce the retrograde response under appropriate conditions, resulting in increased replicative lifespan (RLS). Although the retrograde pathway has been extensively elaborated, the nature of the mitochondrial signal triggering this response has not been clear. Mitochondrial membrane potential (MMP) was severely reduced in $\mathrm{rho}^{0}$ compared to rho ${ }^{+}$cells, and RLS was concomitantly extended. To examine the role of MMP in the retrograde response, MMP was increased in the rho ${ }^{0}$ strain by introducing a mutation in the ATP1 gene, and it was decreased in rho ${ }^{+}$cells by deletion of COX4. The ATP1-111 mutation in rho ${ }^{0}$ cells partially restored the MMP and reduced mean RLS to that of rho ${ }^{+}$cells. COX4 deletion decreased MMP in rho cells to a value intermediate between rho ${ }^{+}$and rho $^{0}$ cells and similarly increased RLS. The increase in expression of CIT2, the diagnostic gene for the retrograde response, seen in rho ${ }^{0}$ cells, was substantially suppressed in the presence of the ATP1-111 mutation. In contrast, CIT2 expression increased in rho ${ }^{+}$cells on deletion of COX4. Activation of the retrograde response results in the translocation of the transcription factor Rtg3 from the cytoplasm to the nucleus. Rtg3-GFP translocation to the nucleus was directly observed in $\mathrm{rho}^{\circ}$ and $\mathrm{rho}^{+} \operatorname{cox} 4 \Delta$ cells, but it was blunted in rho ${ }^{0}$ cells with the ATP1-111 mutation. We conclude that a decrease in MMP is the signal that initiates the retrograde response and leads to increased RLS.

Keywords: Saccharomyces cerevisiae, replicative lifespan, mitochondrial membrane potential, retrograde signaling

\section{INTRODUCTION}

Yeast replicative lifespan (RLS) has been studied extensively to understand genetic determinants of lifespan and the mechanisms of aging. Aging is a complicated multifactorial process and a number of major determinants have been described. These include genome stability, metabolism, stress resistance, and chromatindependent gene regulation, in addition to random (stochastic) events (Jazwinski, 1999, 2005). We have previously demonstrated one mechanism of lifespan determination that results from mitochondrial dysfunction. This mechanism, termed the retrograde response, is a form of inter-organelle signaling that impacts nuclear gene expression, compensates for the loss of tricarboxylic acid cycle intermediates, and increases RLS (Kirchman et al., 1999; Liu and Butow, 1999). Deletion of mitochondrial DNA activates this mechanism (Kirchman et al., 1999), but it also appears to function during normal yeast aging during which the extent of activation of the retrograde response is commensurate with accumulating mitochondrial dysfunction (Borghouts et al., 2004).

The retrograde response is a programmed pathway. The Rtg2 protein responds to a signal generated by dysfunctional mitochondria. Rtg2 then binds to Mks1 which releases its inhibition of the Rtg1-Rtg3 transcription factor, allowing its translocation to the nucleus (Sekito et al., 2000; Dilova et al., 2002, 2004). The Rtg1Rtg3 transcription factor binds to the sequence GTCAC in the target genes' promoters resulting in increased expression of these genes (Liao and Butow, 1993; Rothermel et al., 1997). However, the initial event signaling mitochondrial dysfunction is not known.
On the surface, there seem to be three possible mitochondrial signals that could signal the retrograde response. The first is a drop in the concentration of ATP due to a loss of respiratory function. The second could be an increase in reactive oxygen species (ROS) generated by dysfunctional mitochondria. The third mechanism is a loss of mitochondrial membrane potential (MMP). Loss of MMP is an attractive possibility because aging yeast cells exhibit reduced MMP and activate the retrograde response (Lai et al., 2002; Borghouts et al., 2004). Furthermore, lowering of MMP with the uncoupler dinitrophenol has been shown to increase RLS in yeast (Barros et al., 2004). In this study, we altered MMP in rho ${ }^{0}$ cells by introducing the mutant ATP1-111 which results in a hyperactive Atp1 subunit of the mitochondrial $\mathrm{F}_{1}$-ATPase and an increase in MMP (Francis et al., 2007; Veatch et al., 2009). In contrast, we induced a decrease in MMP in rho ${ }^{+}$cells by deletion of $C O X 4$, which encodes a subunit of cytochrome oxidase. In this way, MMP was altered independent of the presence or absence of mitochondrial DNA. These genetic manipulations facilitate alteration of MMP over the course of a lifespan without the need to replenish chemical agents.

\section{MATERIALS AND METHODS STRAINS AND GROWTH CONDITIONS}

For lifespan analyses and preparation of RNA, yeast cells were cultured at $30^{\circ}$ in YPD (2\% peptone, $1 \%$ yeast extract, $2 \%$ glucose) or YPG (2\% peptone, $1 \%$ yeast extract, $2 \%$ glycerol), as indicated. For some experiments, yeast cells were cultured in SC 
medium $(0.67 \%$ yeast nitrogen base without ammonium sulfate and amino acids, $2 \mathrm{~g} / \mathrm{l}$ complete amino acid drop-out mix, and 2\% glucose). For selection of transformants, cells were cultured on SC medium containing all required nutrients except those needed for selection. The strains used in this study were as follows: YPK9 (MATa, ade2-101 ${ }^{\text {ochre }}$ his3- $\Delta 200$ leu2- $\Delta 1$ lys2-801 $1^{\text {amber }}$ trp1- $\Delta 63$ ura3-52 $\left[\mathrm{rho}^{+}\right]$), described previously (Kirchman et al., 1999) and provided by P.A. Kirchman, Florida Atlantic University, Jupiter, FL; YJR2 (MATa, ade2-101 ${ }^{\text {ochre }}$ his3- $\Delta 200$ leu2- $\Delta 1$ lys2-801 ${ }^{\text {amber }}$ $\operatorname{trp1}-\Delta 63$ ura3-52 $\left.\left[r h o^{0}\right]\right)$, an ethidium bromide-induced rho ${ }^{0}$ derived from YPK9 as follows. Wild type cells (YPK9) were grown in YPD medium in presence of $25 \mu \mathrm{g} / \mathrm{ml}$ ethidium bromide, overnight at $30^{\circ} \mathrm{C}$. Colonies were selected for zero growth on YPG plates and further characterized for loss of mitochondrial DNA by $4^{\prime}$,6-diamidino-2-phenylindole (DAPI) staining. YSK365 phb1 $\Delta$ is a rho ${ }^{0}$ derivative of YPK9 (Kirchman et al., 2003).

\section{PLASMIDS AND TRANSFORMANTS}

All restriction endonucleases were purchased from New England Biolabs Inc., Ipswich, MA, USA. Replacement of the ATP1 gene with the ATP1-111 mutation was accomplished by use of a plasmid based on pRS316 containing the mutated gene sequence (Francis et al., 2007) and was a kind gift from Peter Thorsness (Department of Molecular Biology, University of Wyoming, Laramie, WY, USA). The ATP1-111 insert was sequenced to insure that it contained the necessary mutation and that no other functional mutations in the gene sequence were present. The ATP1-111 insert was excised using Bam $\mathrm{H} 1$ and $\mathrm{Xbal}$ and ligated into pRS306 for integration. The ATP1 insert was cut once with Pfm1, transformed into YJR2 competent cells and selected for growth on plates lacking uracil. $U R A 3$ positive clones were grown overnight in medium lacking uracil and then 5-6h in YPD. The cultures were then plated on YPD plates containing 5-fluoroorotic acid (FOA, $1 \mathrm{mg} / \mathrm{ml}, \mathrm{ZYMO}$ Research). The colonies positive for growth on FOA were grown overnight and DNA isolated (Master Pure Yeast DNA Purification Kit, Epicentre Biotechnologies, Madison, WI, USA). The ATP1-111 sequence was amplified by PCR, cloned into a plasmid vector (Zero Blunt TOPO PCR Cloning kit, Invitrogen, Carlsbad, CA, USA), and sequenced. Transformants containing the correct sequence were analyzed further. Deletion strategies for RTG2, RTG3, and COX4 have been previously described (Kirchman et al., 1999).

Strains containing the RTG3-GFP fusion construct were made from RTG3 deletion strains containing the URA3 marker. The plasmid pRS416-RTG3-GFP (TSB2-71; Sekito et al., 2000) was a kind gift from Zhengchang Liu (University of New Orleans, New Orleans, LA, USA). The insert containing flanking regions was excised using Bam $\mathrm{H} 1$ and $\mathrm{Xba} 1$ and used for transformation. Positive transformants were selected using FOA, as described above, and confirmed by fluorescence microscopy.

\section{RNA ISOLATION AND REVERSE TRANSCRIPTION REAL TIME POLYMERASE CHAIN REACTION (RT-qPCR)}

CIT2 expression was determined in exponentially growing cells at a density of $5-6 \times 10^{7}$ cells $/ \mathrm{ml}$. Cells were grown in YPD and cell densities determined by hemocytometer counts. Cells were centrifuged at $500 \times g$, rinsed once in ice-cold sterile water and snap frozen in liquid nitrogen. RNA was isolated using the hot-acid phenol method (Ausubel et al., 1993) and further purified using the RNeasy Mini Kit (Qiagen Inc., Valencia, CA, USA) with on column DNAase digestion. Final concentration of RNA was determined using a nano-drop 2000 spectrophotometer (Thermo Scientific, Wilmington, DE, USA). Reverse transcription of the RNA was performed using Applied Biosystems TaqMan reverse transcription reagents (Life Technologies Corporation, Carlsbad, CA, USA). Quantitative PCR was performed on an ABI 7300 Real Time PCR system using forward primer: 5'CGAAATCTACCCCATCCATG3' and reverse primer: 5'TCCC ATACGCTCCCTGGAATA3'. CIT2 expression was normalized to expression of $A C T 1$ amplified using forward primer: $5^{\prime}$ TTC CATCCAAGCCGTTTTGT3' ${ }^{\prime}$ and reverse primer: 5'CAGCGTAAA TTGGAACGACGT3'. Relative expression of three additional retrograde target genes was measured. The forward and reverse primers for $I D H 1, I D H 2$, and $A C O 1$ were respectively: 5'TTGACGTATCGTCCATCATTGTC $3^{\prime}$ and $5^{\prime}$ GCCTAAGATGGT ACCGTACATTGA3'; 5'GCCCTATCTTTGTCAACGGATT3' and 5'CAACCAGGTTTTTTGTGATAGATTGT3' ; 5'CCAAGGTATCA AGTGGGTTGTTATT3' and 5'CGAAACCGCCCAAGAATCT3'.

\section{FLOW CYTOMETRY}

Flow cytometry was performed at the Tulane Cancer Center Cell Analysis Core Facility on a BD LSR II multiple laser analyzer. MMP was determined by growing cells overnight to mid$\log$ density $\left(5-6 \times 10^{7}\right.$ cells/ml $)$ in YPD and incubating aliquots with $3^{\prime}, 3^{\prime}$-dihexyloxacarbocyanine $\left(\operatorname{DiOC}_{6}(3)\right)$ (Invitrogen) for $20 \mathrm{~min}$ at $30^{\circ}$. An aliquot was also incubated separately with carbonyl cyanide m-fluoro phenyl hydrazone (FCCP) (SigmaAldrich, St. Louis, MO, USA) for $10 \mathrm{~min}$ before addition of DiOC $_{6}(3)$ to collapse the membrane potential and serve as a control. The cells were centrifuged and washed once with ice-cold Dulbecco's Phosphate Buffered Saline (dPBS; 137.93 mM sodium chloride, $8.06 \mathrm{mM}$ sodium phosphate dibasic, $1.47 \mathrm{mM}$ potassium phosphate monobasic, $2.67 \mathrm{mM}$ potassium chloride, $0.901 \mathrm{mM}$ calcium chloride, and $0.493 \mathrm{mM}$ magnesium chloride) and resuspended in ice-cold YPD before analysis. Mitochondrial mass was determined by incubating an aliquot of the cells with Mitotracker Green (MTG) (Molecular Probes-Invitrogen, Carlsbad, CA, USA) for $35 \mathrm{~min}$. MTG has been reported to selectively enter and stain mitochondria independent of the MMP (Pendergrass et al., 2004, and personal observation). The cells were centrifuged, washed once in ice-cold dPBS, and re-suspended in ice-cold YPD for analysis.

\section{DECONVOLUTION MICROSCOPY}

Microscopy of green fluorescent protein (GFP) expressing cells was performed using a Delta Vision deconvolution epi-fluorescence microscope (Applied Precision, Issaquah, WA, USA) equipped with a CoolSnap HQ2 CCD camera and differential interference contrast (DIC) optics. Cells were grown to mid-log phase in SC medium containing $50 \mathrm{ng} / \mathrm{ml}$ DAPI and embedded in low temperature gelling agarose prior to imaging. Typically 10-12 optical sections of $0.4 \mu \mathrm{m}$ thickness were obtained for the fluorescence images. The optical sections were deconvoluted and sum projected to produce the final image. 


\section{REPLICATIVE LIFESPAN}

Replicative lifespan analyses were performed (Egilmez and Jazwinski, 1989) by spotting $10 \mu \mathrm{l}$ of logarithmically growing cells from liquid YPD or YPG onto YPD plates ( $2 \%$ agar). Individual unbudded cells were then separated from the population by micromanipulation and allowed to produce buds. These buds (virgin cells) were removed and used as the starting population for RLS analysis. Each determination consisted of 40 virgin cells. For each successive bud removed from these cells, they were counted one generation older. Cells were grown at $30^{\circ}$ during the day and at $8.0^{\circ}$ overnight for one generation. Growth at low temperature does not affect RLS (Muller et al., 1980).

\section{EXTRACHROMOSOMAL RIBOSOMAL DNA CIRCLE DETECTION}

Yeast strains were grown overnight in $5 \mathrm{ml}$ YPAD (YPD containing $120 \mu \mathrm{g} / \mathrm{ml}$ adenine). DNA was isolated using the spheroplast method (Lai et al., 2002). Three micrograms of DNA was digested with SpeI. This enzyme does not cut in the rDNA, but releases a 4.1$\mathrm{kb}$ fragment of the $A C T 1$ gene, which was used for quantification. The DNA was electrophoresed on a $0.7 \%$ agarose gel at $1.2 \mathrm{~V} / \mathrm{cm}$ for $18 \mathrm{~h}$ and then transferred to a nylon membrane, which was hybridized with ACT1 and 35S-rDNA probes. The 35S probe was generated from plasmid pCB12 (Borghouts et al., 2004) digested with EcoRI. The ACT1 probe was generated by PCR from genomic DNA of Saccharomyces cerevisiae. Restriction digested and PCR product were purified by electrophoresis, and labeled with $[\alpha-$ ${ }^{32} \mathrm{P}$ ] dCTP, using the RediPrime kit (GE Healthcare, Piscataway, NJ, USA). Blots were scanned using the Typhoon phosphorimager (GE Healthcare, Piscataway, NJ, USA), and quantification was performed using ImageQuant version 5.1 software (Molecular Dynamics, Sunnyvale, CA, USA).

\section{STATISTICS}

Tests of statistical significance were performed using ANOVA or unpaired Student's $t$-test (Statmost 3.0, Data Most Corporation, Sandy, UT, USA). Tukey's post hoc analysis was applied where appropriate (SPSS, IBM Corporation, Amonk, NY, USA). Statistical analyses of life spans were performed using the non-parametric Mann-Whitney test. $P$-values $\leq 0.05$ (two-sided) were considered statistically significant.

\section{RESULTS}

\section{RESTORATION OF MMP IN RHO ${ }^{\circ}$ CELLS AND ATTENUATION IN RHO ${ }^{+}$ CELLS}

We first examined the MMP in $\mathrm{rho}^{0}$ and rho ${ }^{+}$cells. Figure 1A shows a DiOC $_{6}$ flow cytometry labeling experiment. $\mathrm{DiOC}_{6}$ is taken up by mitochondria in an MMP-dependent manner. Collapse of the MMP by administration of FCCP prevents this uptake and provides a measure of background fluorescence. When corrected for $\mathrm{DiOC}_{6}$ uptake in the presence of FCCP and normalized for mitochondrial mass as determined by MTG staining, rho $^{0}$ cells had an average of only $14 \%$ of the MMP compared to rho ${ }^{+}$. In rho ${ }^{0}$ cells with the ATP1-111 mutation (Figure 1B), the MMP was partially restored. However, these cells were still unable to grow on glycerol, indicating inability to respire. MMP was also reduced in $\mathrm{rho}^{+} \operatorname{cox} 4 \Delta$ cells compared to $\mathrm{rho}^{+}$, but not to the level of the rho ${ }^{0}$ cells (Figure 1B). A comparison of the effects of these genetic manipulations on MMP after normalization to mitochondrial mass in this representative experiment is shown in Figure 1C.

\section{EFFECT OF MANIPULATING THE MMP ON CIT2 EXPRESSION}

We then determined whether increasing MMP in rho ${ }^{0}$ cells interferes with retrograde response signaling by measuring CIT2 expression. An increase in CIT2 expression is considered diagnostic for the activation of the retrograde response (Liao and Butow, 1993). As shown in Figure 2, CIT2 expression in rho ${ }^{0}$ cells is almost threefold higher than in $\mathrm{rho}^{+}$cells. Increasing the MMP in rho $^{0}$ cells in the presence of the ATP1-111 mutation significantly reduced CIT2 expression, suggesting that a decrease in MMP is the initial signal for the retrograde response. CIT2 expression was significantly higher in the $\mathrm{rho}^{+} \operatorname{cox} 4 \Delta$ strain compared to the rho $^{+}$control (Figure 2), which we would expect since MMP is lower as shown in Figure 1. CIT2 expression was also measured in the rho ${ }^{0}$ ATP1-111 mutant containing deletions of either RTG2 or $R T G 3$, both of which are transducers of the retrograde response. CIT2 expression was very low in both cases (Figure 2). This was also the case when these genes were deleted in the rho ${ }^{+} \operatorname{cox} 4 \Delta$ strain (Figure 2). These results demonstrate that the activation of CIT2 depends on retrograde signaling in these strains. The effects of altering MMP on CIT2 expression were recapitulated by three other retrograde response target genes, $I D H 1, I D H 2$, and $A C O 1$ (Figure A1 in Appendix).

\section{Rtg3 TRANSLOCATION TO THE NUCLEUS IS DEPENDENT ON MMP}

The translocation of the Rtg1-Rtg3 transcription factor from the cytoplasm to the nucleus is an integral feature of the retrograde response, and it is necessary for the induction of retrograde response target genes such as CIT2. We therefore examined the intracellular localization of the Rtg3 protein fused to GFP in the yeast strains in which we manipulated the MMP. This construct has been used previously to monitor Rtg1-Rtg3 translocation, and it is active in supporting induction of CIT2 expression (Sekito et al., 2000). Figure 3A shows deconvolution micrographs of cells containing the RTG3-GFP fusion in place of endogenous $R T G 3$. In rho ${ }^{+}$cells at mid-log growth phase, the Rtg3-GFP protein showed a diffuse, cytoplasmic fluorescence (YPK9). This is in contrast to the rho ${ }^{0}$ cells (YJR2), in which the Rtg3-GFP was seen mainly in the nucleus, as indicated by the colocalization of the GFP fluorescence with the fluorescence of DAPI which binds to the nuclear DNA. When we imaged the rho ${ }^{0}$ ATP1-111 cells, the Rtg3-GFP localization resembled that in $\mathrm{rho}^{+}$cells, indicating that Rtg3 is not translocated to the nucleus in these cells. In the cox $4 \Delta$ cells, Rtg3 translocation was intermediate to that in the rho ${ }^{+}$and rho $^{0}$ cells, consistent with the partial activation of the retrograde response indicated by CIT2 expression (Figure 2). These results are quantified in Figure 3B. Thus, the localization of Rtg3 is dependent on MMP and consistent with the activation of CIT2 expression.

\section{RLS EXTENSION DEPENDS ON MMP}

Activation of the retrograde response in rho $^{0}$ cells extends RLS (Kirchman et al., 1999). If this activation depends on MMP, we should observe predictable effects on RLS by manipulating 


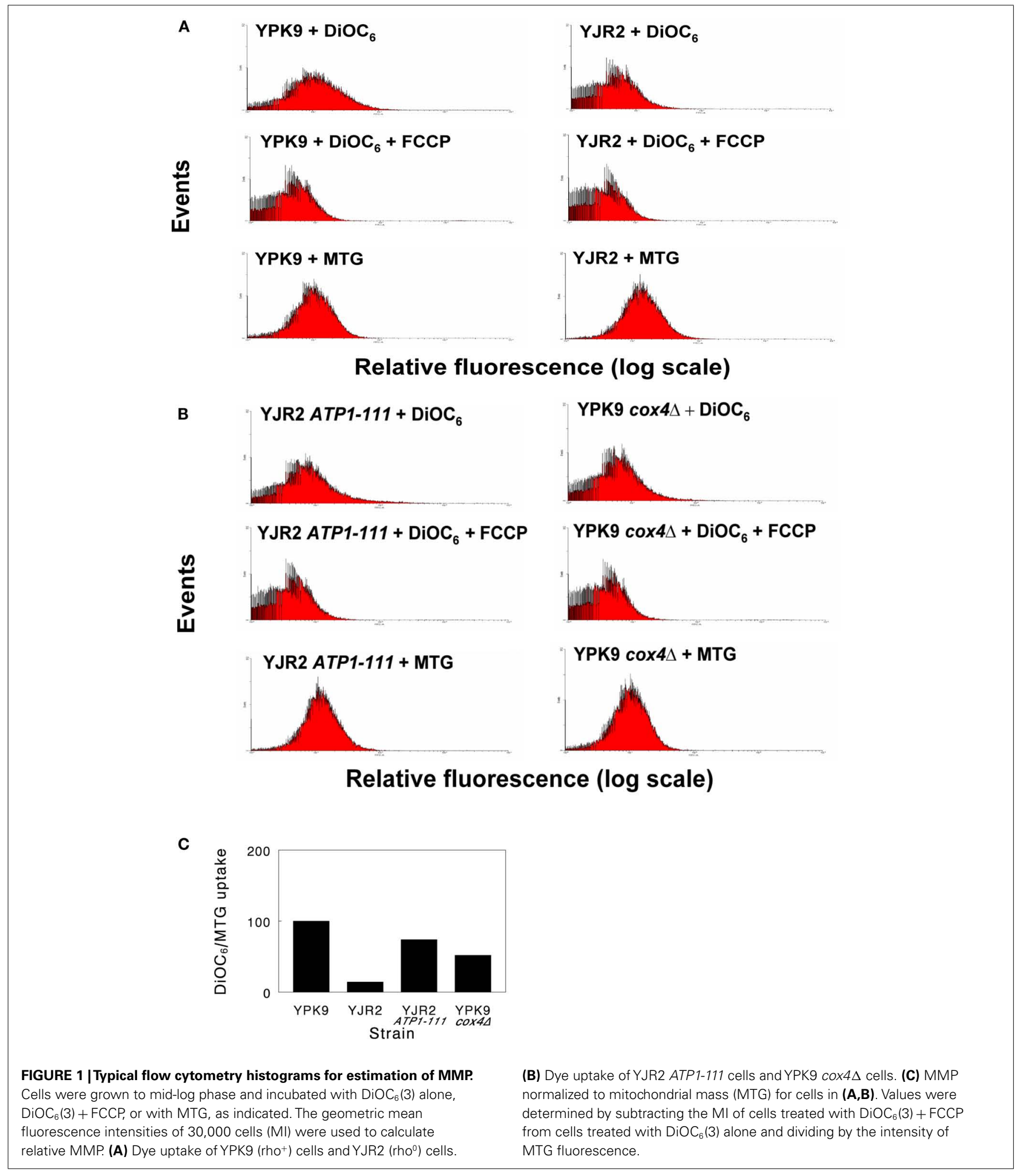

the MMP. As shown in Figure 4A, RLS in the rho ${ }^{0}$ cells was increased substantially, as previously reported (Kirchman et al., 1999). However, the ATP1-111 mutation completely suppressed the RLS extension. In fact, the RLS was not significantly different than the RLS in rho ${ }^{+}$cells. This result supports the conclusion that reduced MMP in rho ${ }^{0}$ cells signals the retrograde response resulting in the increase in RLS. Interestingly, the lifespan extension seen in the rho ${ }^{0}$ strain is almost completely abolished even though the 


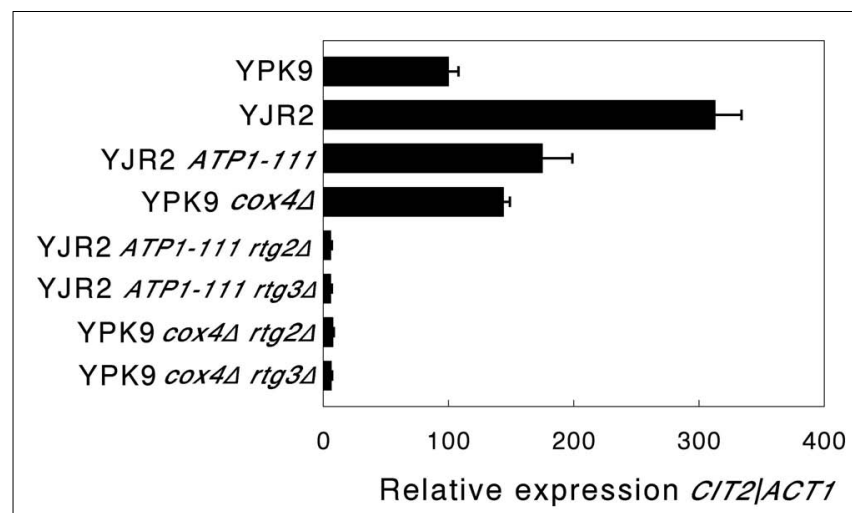

FIGURE 2 | CIT2 expression. Expression of CIT2 relative to ACT1 was determined by qRT-PCR as described in Section "Materials and Methods." The strains used were as follows: YPK9 $\left(\mathrm{rho}^{+}\right)$; YJR2 (rho $\left.{ }^{\circ}\right)$; YJR2 ATP1-111;

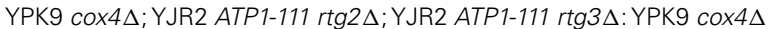
$r \operatorname{tg} 2 \Delta ;$ YPK9 $\operatorname{cox} 4 \Delta r \operatorname{tg} 3 \Delta$. Error bars are \pm SEM for $N=3$ or 4 determinations. $P$-values adjusted for multiple comparisons are $P<0.0002$ for YPK9 vs. YJR2; $P<0.05$ for YJR2 vs. YJR2 ATP1-111; $P<0.02$ for YPK9 vs. YPK9 $\operatorname{cox} 4 \Delta$

MMP is not fully restored, indicating that there must be some threshold reduction in MMP for the retrograde response and RLS extension to be triggered. To provide further support for the role of MMP in the retrograde response, we measured RLS in the rho ${ }^{+}$ $\operatorname{cox} 4 \Delta$ strain that has a lower MMP than the rho ${ }^{+}$strain but maintains its mitochondrial DNA. As shown above in Figure 1C, deletion of COX4 in $\mathrm{rho}^{+}$cells resulted in an MMP intermediate to that of the rho ${ }^{+}$and rho ${ }^{0}$ cells. The RLS was significantly increased in the COX4 deletion strain also intermediate to that of the $\mathrm{rho}^{+}$and $\mathrm{rho}^{0}$ strains (Figure 4B). This increase in RLS was due to activation of the retrograde response, as deletion of either RTG2 or RTG3 abrogated the RLS extension (Figure 4B). Thus, the activation of the retrograde response and extension of RLS is dependent on MMP.

\section{ERC ACCUMULATION IN RHO ${ }^{\circ}$ CELLS IS DEPENDENT ON MMP AND THE MITOCHONDRIAL NUCLEOID}

Extrachromosomal ribosomal DNA circle (ERC) levels are higher in rho $^{0}$ as compared to rho ${ }^{+}$cells, and they accumulate with replicative age (Conrad-Webb and Butow, 1995; Sinclair and Guarente, 1997; Borghouts et al., 2004). We previously showed that ERC production in $\mathrm{rho}^{0}$ cells involves Rtg2 which cannot suppress their accumulation when it is engaged in retrograde signaling (Borghouts et al., 2004). We therefore expected that increasing MMP in rho ${ }^{0}$ cells would reduce $\mathrm{Rtg} 2$ participation in retrograde signaling and suppress formation of ERC. ERC accumulation was significantly increased in the rho ${ }^{0}$ as compared to the rho ${ }^{+}$ strain (Figure 5). Elevation of MMP in the ATP1-111 rho $^{0}$ strain reduced ERC accumulation to a level approaching that found in the $\mathrm{rho}^{+}$cells. Interestingly, ERC levels in the cox $4 \Delta \mathrm{rho}^{+}$ strain were the same or lower than in the rho ${ }^{+}$cells. This suggests that defects in, or the absence of the mitochondrial nucleoid itself in addition to MMP decrease are necessary to trigger ERC formation.

\section{A FREE RADICAL SCAVENGER DOES NOT AFFECT ACTIVATION OF THE RETROGRADE RESPONSE}

Mitochondrial membrane potential changes are associated with ROS production by mitochondria (Gourlay et al., 2004). Interruption of the electron transport chain at any point results in increased ROS in yeast, the levels of which depend on the exact nature of the disruption (Barros et al., 2003). Thus, ROS could be downstream of MMP in retrograde response signaling. We used the free radical scavenger and antioxidant phenyl tert butyl nitrone (PBN) and measured ROS by fluorescence microscopy, using the peroxide-sensitive dye $2^{\prime}, 7^{\prime}$-dichlorodihydrofluorescein diacetate $\left(\mathrm{H}_{2}\right.$ DCFDA $)$. ROS were nearly undetectable in the four strains used in this study. We previously reported that YSK365 phb1s cells produce ample ROS under normal growth conditions (Kirchman et al., 2003). PBN substantially quenched ROS production at $0.1 \mathrm{mg} / \mathrm{ml}$ in YSK365 phb1 $\Delta$ cells (Figure 6A), confirming its ROS-scavenging effect. As shown in Figure 6B, increasing concentrations of PBN had no effect on CIT2 expression, indicating that ROS do not appear to play a role in retrograde response activation. Quenching ROS also did not block RLS extension in rho ${ }^{0}$ cells, indicating that the activation of the retrograde response and concomitant extension of RLS by decreased MMP is not mediated by ROS (Figure 6C).

\section{DISCUSSION}

We have shown that a decrease in MMP is the event proximal to the mitochondrion that signals the retrograde response. Elevation of MMP in rho ${ }^{0}$ cells possessing the ATP1-111 mutation (Figure 1) suppressed translocation of the Rtg3 transcription factor from the cytoplasm to the nucleus (Figure 3) and induction of CIT2 expression (Figure 2), a diagnostic of the retrograde response. Furthermore, it suppressed the extension of RLS seen in rho ${ }^{0}$ cells possessing wild type ATP1 (Figure 4). In contrast, reduction of MMP in $\mathrm{rho}^{+}$cells possessing the COX4 deletion induced the translocation of Rtg3 to the nucleus (Figure 3) and expression of CIT2 (Figure 2), while increasing the RLS (Figure 4). There is an inverse relationship between MMP and retrograde response activation, as measured by CIT2 induction. However, CIT2 expression levels, unlike translocation of Rtg3, are not directly proportional to RLS extension. This suggests that in addition to the MMP there are other signals generated by mitochondria that impinge on the retrograde response and affect RLS. It should also be noted that expression of CIT2 itself does not determine RLS (Kirchman et al., 1999).

One candidate for the additional signal impacting the retrograde response is the Ras2 pathway. RAS2 is known to be necessary for activation of the retrograde response in terms of both CIT2 expression and RLS extension (Kirchman et al., 1999). This Ras2 signaling is likely to be cAMP-independent (Sun et al., 1994). Ira1 and Ira2 are components of the mitochondrial proteome in yeast (Sickmann et al., 2003). These GTPase-activating proteins negatively regulate Ras2, and it is known that deletion of IRA2 either alone or with IRA1 causes constitutively high Ras2-GTP loading (Colombo et al., 2004). It has also been shown that glucose phosphorylation enhances Ras2-GTP loading, likely through inhibition of the Ira proteins (Colombo et al., 2004). Whether differences in signaling through Ras2 due to loss of all or most 


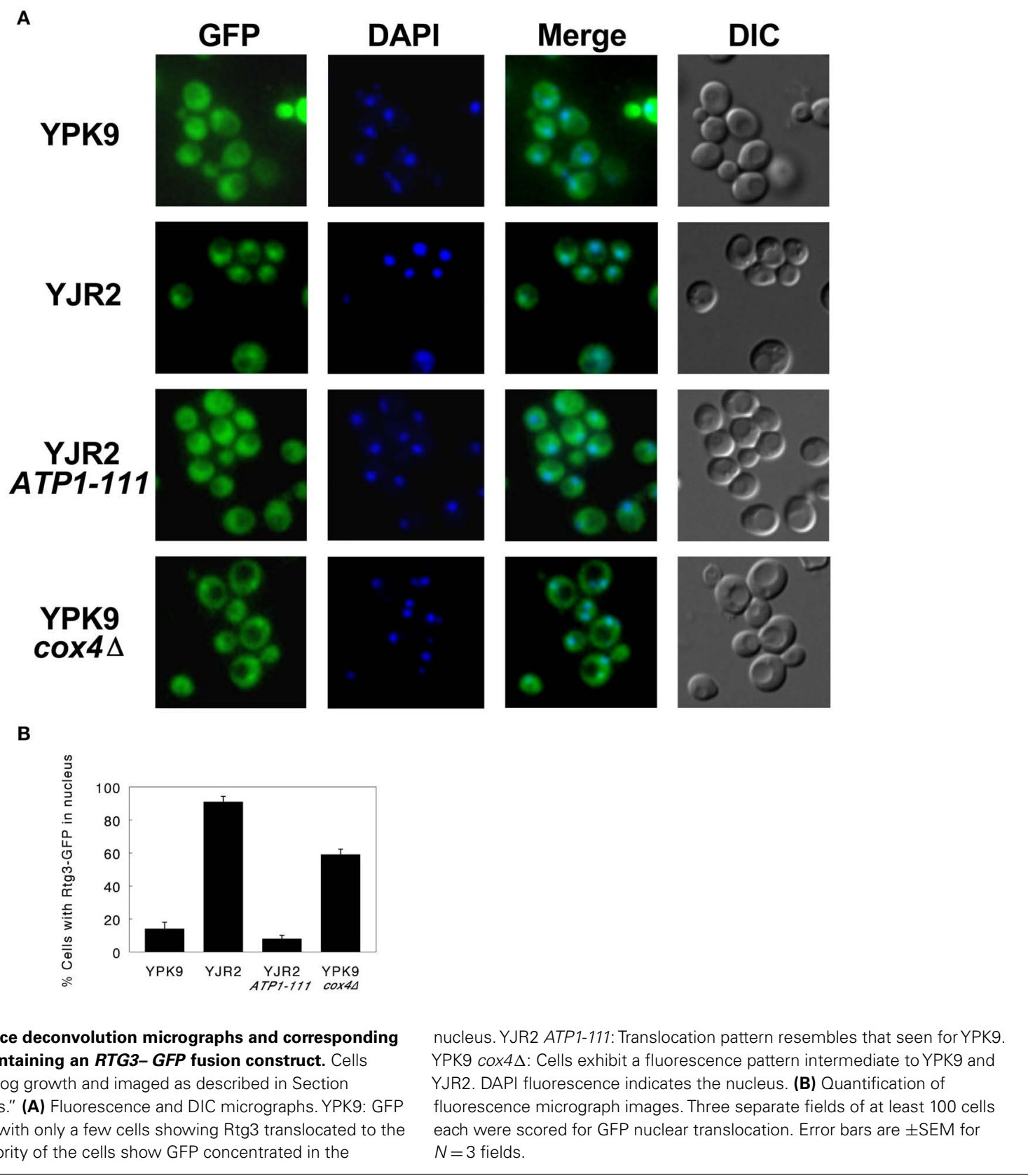

FIGURE 3 | Fluorescence deconvolution micrographs and corresponding DIC images of cells containing an RTG3- GFP fusion construct. Cells were harvested at mid-log growth and imaged as described in Section "Materials and Methods." (A) Fluorescence and DIC micrographs. YPK9: GFP fluorescence is diffuse with only a few cells showing Rtg3 translocated to the nucleus. YJR2: The majority of the cells show GFP concentrated in the of the mitochondrial DNA are necessary for full activation of the retrograde response awaits further studies.

The nutritional status-responsive TOR pathway modulates the retrograde response (Komeili et al., 2000; Liu et al., 2003), and it has been shown that TOR 1 is involved in RLS extension by nutrient limitation in yeast (Kaeberlein et al., 2005). As shown recently, the downstream target genes of the retrograde response and nutrient limitation, whether through reduction of glucose or non-essential amino acids, are very similar (Wang et al., 2010), even though the signaling pathways differ (Jiang et al., 2000). The common denominator here may be the TOR pathway (Jazwinski, 2012). Interestingly, TOR1 has been implicated in another signaling pathway from the mitochondrion to the nucleus, called mitochondrial back-signaling that extends RLS (Heeren et al., 2009). Recently, it has been found that defects in the mitochondrial translation control machinery also extend RLS by reduction of TOR signaling concomitant with disruption of respiration (Caballero et al., 2011). Besides the involvement of TOR, all of these pathways share in common mitochondrial defects that result in respiratory dysfunction.

Our analysis of ERC accumulation is also informative with regard to the complexity of the retrograde response. There was an increase in ERC accumulation in rho ${ }^{0}$ cells (Figure 5), as expected, and this was significantly reduced by the ATP1-111 mutation. 


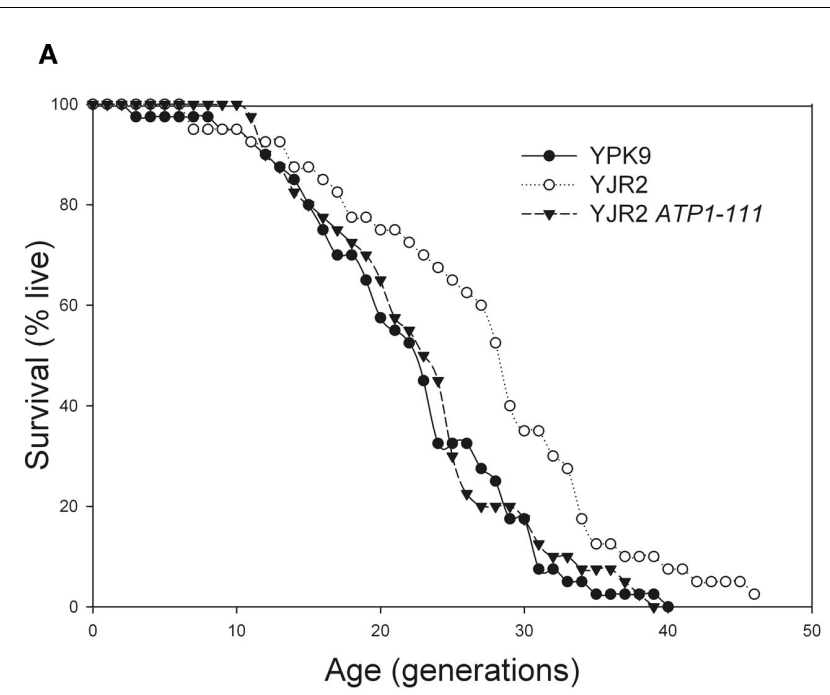

B

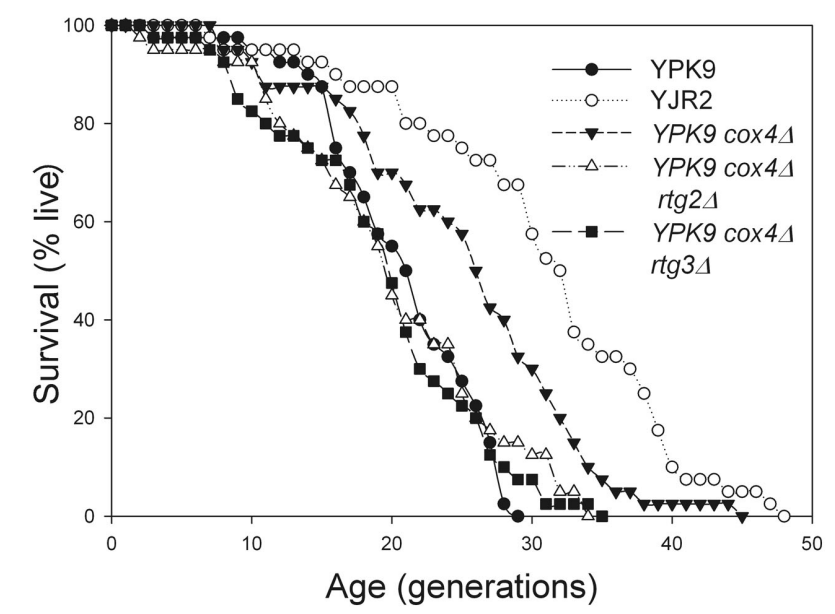

FIGURE 4 | Replicative lifespan determinations. (A) YPK9, YJR2, and YJR2 ATP1-111. Mean life spans: YPK9: 21.2 generations; YJR2: 26.3 generations; YJR2 ATP1-111: 22.0 generations. $P=0.009$ YPK9 vs. YJR2 $P=0.71$ YPK9 vs. YJR2 ATP1-111. (B) YPK9, YJR2, YPK9 cox4 $\Delta$, YPK9 $\operatorname{cox} 4 \Delta r \operatorname{tg} 2 \Delta$, and YPK9 cox4 $\Delta$ rtg3 $\Delta$. Mean life spans:YPK9: 19.8, YJR2: 29.8, YPK9 cox4 $\Delta: 24.1$, YPK9 cox4 $\Delta$ rtg2 $\Delta: 19.1$, and YPK9 $\operatorname{cox} 4 \Delta r \operatorname{rtg} 3 \Delta$ 18.4. $P=1 \times 10^{-6}$ YPK9 vs. YJR2; $P=0.007$ YPK9 vs. YPK9 $\operatorname{cox} 4 \Delta ; P=0.61$ YPK9 vs. YPK9 cox $4 \Delta r t g 2 \Delta ; P=0.39$ YPK9 vs. YPK9 $\operatorname{cox} 4 \Delta r t g 3 \Delta ;$ $P=0.0045$ YJR2 vs. YPK9 cox4 $\Delta$.

This indicates a reduction in retrograde signaling through Rtg2, which suppresses rDNA instability when it is available to do so (Borghouts et al., 2004). However, the rho ${ }^{+} \operatorname{cox} 4 \Delta$ cells, which still contain mitochondrial DNA, have ERC levels similar to rho $^{+}$cells. This indicates some direct effect of the mitochondrial DNA/nucleoid on retrograde response signaling and ERC production. Certain atp2 mutants which retain mitochondrial DNA also do not accumulate ERC, even though they are deficient in MMP (Lai et al., 2002). Perhaps these differences in ERC accumulation reflect the operation of inter-genomic signaling, a cellular response to loss of mitochondrial DNA (Poyton and McEwen, 1996). This inter-genomic signaling response seems to bypass

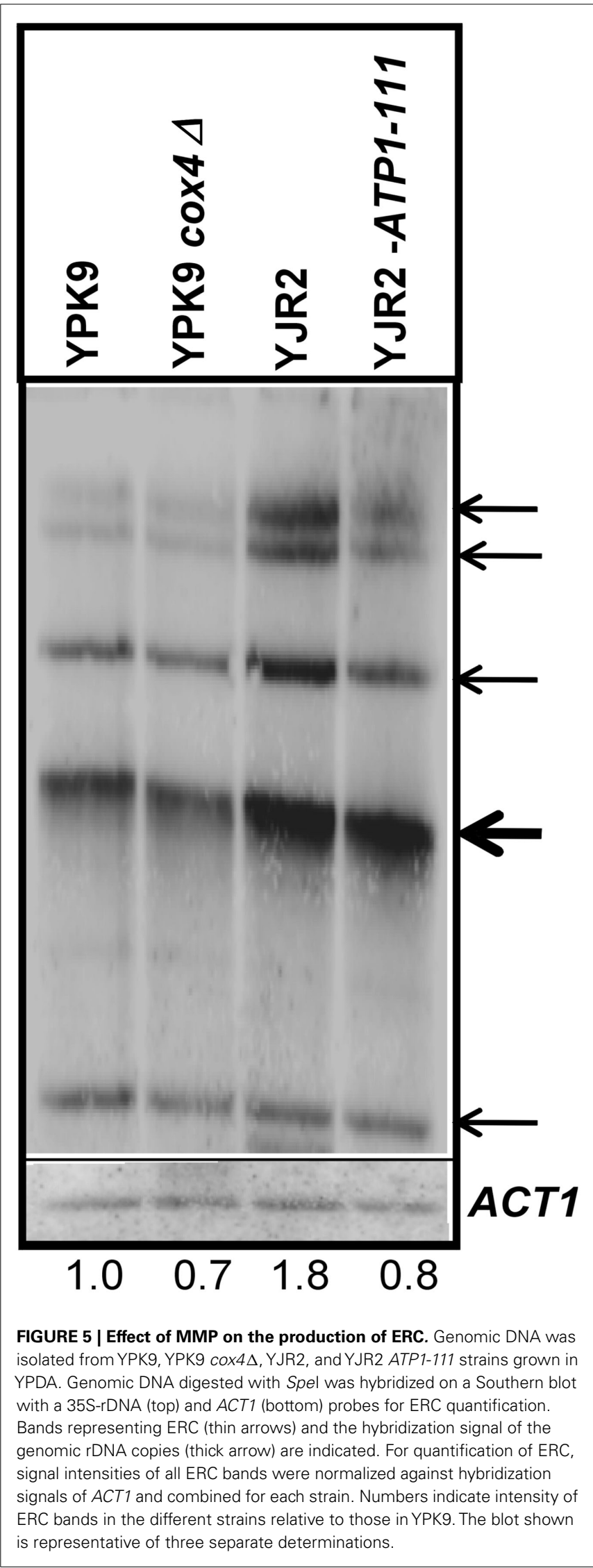




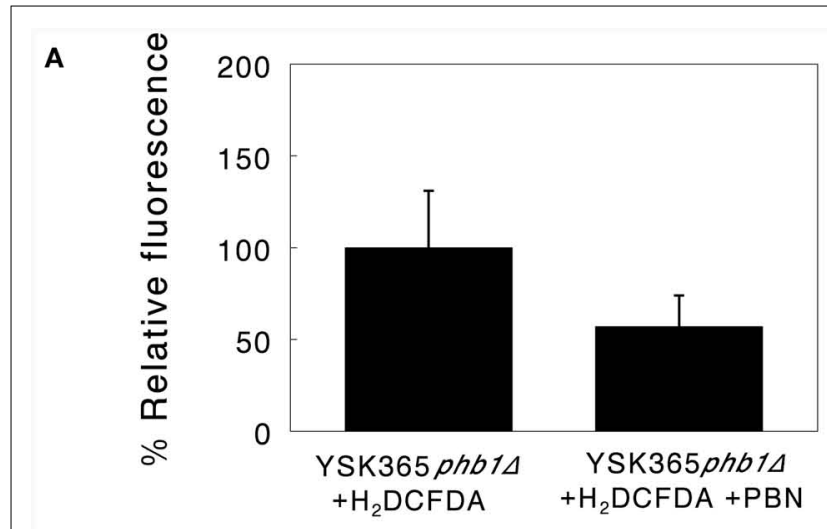

B
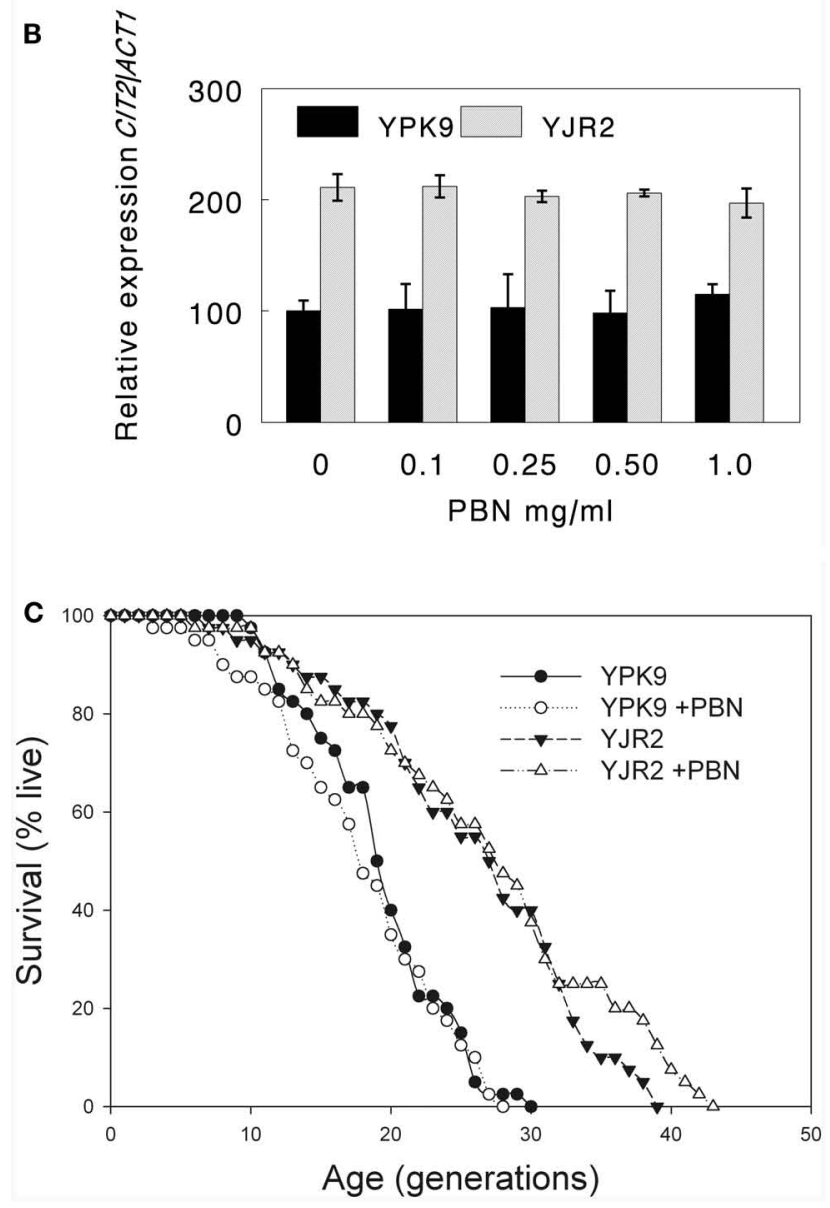

FIGURE 6 | Effect of PBN on ROS, CIT2 expression, and RLS. (A) PBN at $0.1 \mathrm{mg} / \mathrm{ml}$ significantly reduces oxidation of $\mathrm{H}_{2}$ DCFDA. Percent fluorescence intensity is expressed as mean fluorescence intensity per cell area, determined by the SoftWorx analysis software provided with the deconvolution epi-fluorescence microscope. Error bars are \pm SD. $P=0.009$ for YSK365 phb1 $\Delta$ cells grown in the presence or absence of PBN. (B) Effect of increasing concentrations of PBN on C/T2 expression in YPK9 and YJR2 cells. Error bars are \pm SEM for three or four experiments. The effect of PBN concentration on CIT2 expression was not significant for either YPK9 or YJR2 by ANOVA. (C) RLS of YPK9 and YJR2 in the presence of $0.1 \mathrm{mg} / \mathrm{ml}$ PBN. Mean life spans are YPK9: 18.3, YPK9 $+0.1 \mathrm{mg} / \mathrm{ml} \mathrm{PBN:} \mathrm{17.9,} \mathrm{YJR2}$ 25.1; YJR2 $+0.1 \mathrm{mg} / \mathrm{ml} \mathrm{PBN:} \mathrm{26.0.} P=0.0001$ YPK9 vs. YJR2; $P=0.4$ for $Y P K 9 \pm 0.1 \mathrm{mg} / \mathrm{ml} \mathrm{PBN} ; P=0.7$ for YJR2 $\pm 0.1 \mathrm{mg} / \mathrm{ml} P B N$.

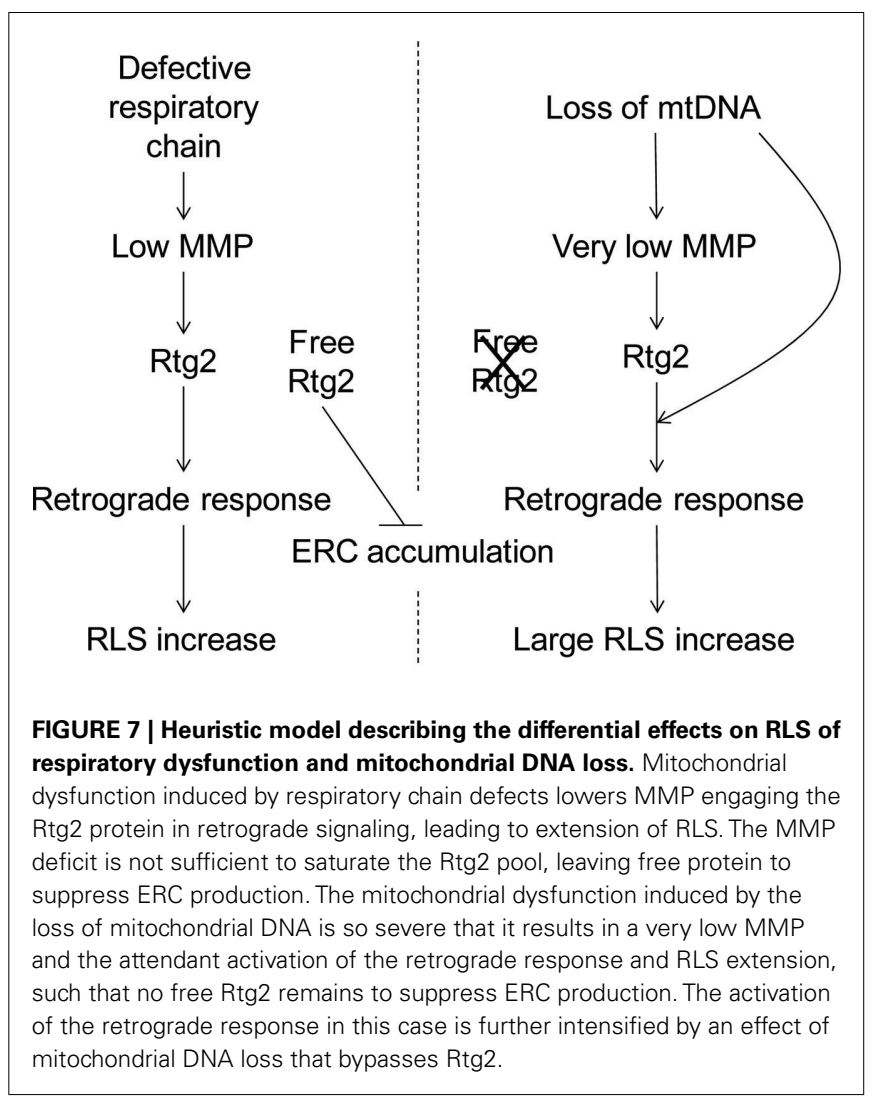

Rtg2 and directly affects Rtg3 in an altered mechanism of RLS extension in rho ${ }^{0}$ cells (Woo and Poyton, 2009). We propose a heuristic model to encompass the effects of loss of mitochondrial DNA together with decrease in MMP on the retrograde response and RLS (Figure 7). This model takes into account the mutually exclusive roles of Rtg2 in retrograde signaling and maintenance of genome stability. It also explains why the effects of attenuated MMP on the retrograde response and RLS extension are different in cells that possess $\left(\mathrm{rho}^{+}\right)$and those that lack $\left(\mathrm{rho}^{0}\right)$ mitochondrial DNA.

Although we have shown here that the MMP is a signal proximal to the mitochondrion that activates the retrograde response, how this signal interacts with the next signal transducer in this pathway, Rtg2, is not clear. Increases in ROS have been proposed as a signal resulting from mitochondrial dysfunction. ROS production increases in yeast with replicative age and may be the cause of apoptotic death at the end of their lifespan (Laun et al., 2001). However, the retrograde signaling examined here does not seem to be due to an increase in ROS. The ROS scavenger PBN did not suppress the induction of CIT2 expression or increase in RLS in rho ${ }^{0}$ cells (Figure 6).

Another possible candidate for the initiator of mitochondriato-nucleus signaling is a decrease in ATP concentration brought on by a dysfunctional respiratory chain. In this scenario, Rtg2 functions as an ATP sensor, which is consistent with the requirement of its ATP-binding domain for its function (Liu and Butow, 2006). In the presence of ATP, Rtg2 would be released from Mks1 which would inhibit retrograde signaling. However direct support for this 
mechanism has not been shown because local ATP concentrations in organelles cannot reliably be measured. It also seems unlikely that rho ${ }^{0}$ cells would have a reduced ATP concentration compared to wild type cells because, in general, ATP concentrations have been shown to be tightly regulated (Goldberg et al., 2009) and rho $^{0}$ cells provided with adequate glucose increase glycolysis to compensate for the lack of ATP production from respiration. In addition, ATP must fuel the hyperactive $\mathrm{F}_{1}$-ATPase in the ATP1111 mutant in rho ${ }^{0}$ cells to raise MMP and suppress the retrograde response, which would lower rather than increase ATP levels. This is inconsistent with the notion that low ATP signals the retrograde response. Furthermore, addition of glucose to aerobically growing yeast cells in a chemostat results in a rapid drop in cellular ATP followed by a recovery to a new steady state (Somsen et al., 2000), indicating that ATP is maintained at relatively constant levels.

The mechanism by which the decline in MMP is coupled to signaling through Rtg2 remains to be resolved. Recently, a tethering complex in yeast between mitochondria and the endoplasmic reticulum which appears necessary for phospholipid exchange and which would facilitate communication between the two compartments has been demonstrated (Kornmann et al., 2009).

\section{REFERENCES}

Ausubel, F. M., Brent, R., Kingston, R. E., Moore, D. D., Seidman, J. G., Smith, J. A., and Struhl, K. (1993). Current Protocols in Molecular Biology. New York: John Wiley \& Sons.

Barros, M. H., Bandy, B., Tahara, E. B., and Kowaltowski, A. J. (2004). Higher respiratory activity decreases mitochondrial reactive oxygen release and increases life span in Saccharomyces cerevisiae. J. Biol. Chem. 279, 49883-49888.

Barros, M. H., Netto, L. E. S., and Kowaltowski, A. J. (2003). $\mathrm{H}_{2} \mathrm{O}_{2}$ generation in Saccharomyces cerevisiae respiratory PET mutants: effect of cytochrome c. Free Radic. Biol. Med. 35, 179-188.

Borghouts, C., Benguria, A., Wawryn, J., and Jazwinski, S. M. (2004). Rtg2 protein links metabolism and genome stability in yeast longevity. Genetics 166, 765-777.

Caballero, A., Ugidos, A., Liu, B., Oling, D., Kvint, K., Hao, X., Mignat, C., Nachin, L., Molin, M., and Nystrom, T. (2011). Absence of mitochondrial translation control proteins extends lifespan by activating sirtuin-dependent silencing. Mol. Cell 42, 390-400.

Colombo, S., Ronchetti, D., Thevelein, J. M., Winderickx, J., and Martegani, E. (2004). Activation state of the Ras2 protein and glucoseinduced signaling in Saccharomyces cerevisiae. J. Biol. Chem. 279, 46715-46722.

Conrad-Webb, H., and Butow, R. A. (1995). A polymerase switch in the synthesis of rRNA in Saccharomyces cerevisiae. Mol. Cell Biol. 15, 2420-2428.

Dilova, I., Aronova, S., Chen, J. C., and Powers, T. (2004). Tor signaling and nutrient-based signals converge on Mks1p phosphorylation to regulate expression of $\mathrm{Rtg} 1 \cdot \operatorname{Rtg} 3 \mathrm{p}$ dependent target genes. J. Biol. Chem. 279, 46527-46535.

Dilova, I., Chen, C. Y., and Powers, T. (2002). Mks1 in concert with TOR signaling negatively regulates RTG target gene expression in $S$. cerevisiae. Curr. Biol. 12, 389-395.

Egilmez, N. K., and Jazwinski, S. M. (1989). Evidence for the involvement of a cytoplasmic factor in the aging of the yeast Saccharomyces cerevisiae. J. Bacteriol. 171, 37-42.

Francis, B. R., White, K. H., and Thorsness, P. E. (2007). Mutations in the Atplp and Atp3p subunits of yeast ATP synthase differentially affect respiration and fermentation in Saccharomyces cerevisiae. J. Bioenerg. Biomembr. 39, 127-144.

Goldberg, A. A., Bourque, S. D., Kyryakov, P., Gregg, C., BoukhViner, T., Beach, A., Burstein, M. T., Machkalyan, G., Richard, V., Rampersad, S., Cyr, D., Milijevic, S., and Titorenko, V. I. (2009). Effect of calorie restriction on the metabolic history of chronologically aging yeast. Exp. Gerontol. 44, 555-571.

Gourlay, C. W., Carpp, L. N., Timpson, P., Winder, S. J., and Ayscough, K. R. (2004). A role for the actin cytoskeleton in cell death and aging in yeast. J. Cell Biol. 164, 803-809.

This communication could involve direct protein linkage between the two compartments or through a gradient of a small molecule such as calcium which could signal mitochondrial dysfunction (Kornmann and Walter, 2010). The retrograde response interacts with the other mechanisms of inter-organelle signaling, as well as caloric restriction pathways as part of a robust and highly regulated program of mitochondria-to-nucleus signaling. How the MMP interacts with these other pathways awaits further study.

\section{ACKNOWLEDGMENTS}

The authors would like to thank Dr. Peter Thorsness (University of Wyoming) for the gift of the plasmid containing the ATP1-111 mutant, Dr. Paul A. Kirchman (Florida Atlantic University) for the yeast strain YPK9, and Dr. Zhengchang Liu (University of New Orleans) for the plasmid pRS416-RTG3-GFP (TSB2-71) containing the RTG3-GFP fusion construct. The authors would also like to acknowledge Mary Price, Beatriz E. Finkel-Jimenez, and the Louisiana Cancer Research Consortium FACS Core for help with the flow cytometry experiments. This work was supported in part by NIH grant AG006168.

Heeren, G., Rinnerthaler, M., Laun, P., von Seyerl, P., Kossler, S., Klinger, H., Hager, M., Bogengruber, E., Jarolim, S., Simon-Nobbe, B., Schuller, C., Carmona-Gutierrez, D., Breitenbach-Koller, L., Muck, C. Jansen-Durr, P., Criollo, A., Kroemer, G., Madeo, F., and Breitenbach, M. (2009). The mitochondrial ribosomal protein of the large subunit, Afolp, determines cellular longevity through mitochondrial back-signaling via TOR1. Aging 1, 622-636.

Jazwinski, S. M. (1999). Molecular mechanisms of yeast longevity. Trends Microbiol. 7, 247-252.

Jazwinski, S. M. (2005). The retrograde response links metabolism with stress responses, chromatindependent gene activation, and genome stability in yeast aging. Gene 354, 22-27.

Jazwinski, S. M. (2012). "The retrograde response and other pathways of interorganelle communication in yeast replicative aging," in Aging Research in Yeast, eds M. Breitenbach, S. M. Jazwinski, and P. Laun (New York, NY: Springer). 79-100.

Jiang, J. C., Jaruga, E., Repnevskaya, M. V., and Jazwinski, S. M. (2000). An intervention resembling caloric restriction prolongs life span and retards aging in yeast. FASEB J. 14, 2135-2137.

Kaeberlein, M., Powers, R. W. III, Steffen, K. K., Westman, E. A., Hu, D. Dang, N., Kerr, E. O., Kirkland, K. T., Fields, S., and Kennedy, B. K. (2005).
Regulation of yeast replicative life span by TOR and Sch9 in response to nutrients. Science 310, 1193-1196.

Kirchman, P. A., Kim, S., Lai, C. Y., and Jazwinski, S. M. (1999). Interorganelle signaling is a determinant of longevity in Saccharomyces cerevisiae. Genetics 152, 179-190.

Kirchman, P. A., Miceli, M. V., West, R. L., Jiang, J. C., Kim, S., and Jazwinski, S. M. (2003). Prohibitins and Ras2 protein cooperate in the maintainance of mitochondrial function during yeast aging. Acta Biochim. Pol. 50, 1039-1056.

Komeili, A., Wedaman, K. P., O'Shea, E K., and Powers, T. (2000). Mechanism of metabolic control: target of rapamycin signaling links nitrogen quality to the activity of the Rtg1 and Rtg3 transcription factors. J. Cell Biol. 151, 863-878.

Kornmann, B., Currie, E., Collins, S. R. Schuldiner, M., Nunnari, J., Weissman, J. S., and Walter, P. (2009). An ER-mitochondria tethering complex revealed by a synthetic biology screen. Science 325, 477-480.

Kornmann, B., and Walter, P. (2010). ERMES-mediated ER-mitochondria contacts: molecular hubs for the regulation of mitochondrial biology. J. Cell Sci. 123(Pt 9), 1389-1393.

Lai, C. Y., Jaruga, E., Borghouts, C., and Jazwinski, S. M. (2002). A mutation in the ATP2 gene abrogates the age asymmetry between mother and daughter cells of the yeast Saccharomyces cerevisiae. Genetics 162 , 73-87. 
Laun, P., Pichova, A., Madeo, F., Fuchs, J., Ellinger, A., Kohlwein, S., Dawes, I., Frohlich, K. U., and Breitenbach, M. (2001). Aged mother cells of Saccharomyces cerevisiae show markers of oxidative stress and apoptosis. Mol. Microbiol. 39, 1166-1173.

Liao, X., and Butow, R. A. (1993). RTG1 and RTG2: two yeast genes required for a novel path of communication from mitochondria to the nucleus. Cell 72, 61-71.

Liu, Z., and Butow, R. A. (1999). A transcriptional switch in the expression of yeast tricarboxylic acid cycle genes in response to a reduction or loss of respiratory function. Mol. Cell. Biol. 19, 6720-6728.

Liu, Z., and Butow, R. A. (2006). Mitochondrial retrograde signaling. Annu. Rev. Genet. 40, 159-185.

Liu, Z., Sekito, T., Spirek, M., Thornton, J., and Butow, R. A. (2003). Retrograde signaling is regulated by the dynamic interaction between $\operatorname{Rtg} 2 \mathrm{p}$ and Mks1p. Mol. Cell 12, 401-411.

Muller, I., Zimmermann, M., Becker, D., and Flomer, M. (1980). Calendar life span versus budding life span of Saccharomyces cerevisiae. Mech. Ageing Dev. 12, 47-52.
Pendergrass, W., Wolf, N., and Poot, M. (2004). Efficacy of MitoTracker Green $^{\mathrm{TM}}$ and CMXRosamine to measure changes in mitochondrial membrane potentials in living cells and tissues. Cytometry $A \quad 61 \mathrm{~A}$, 162-169.

Poyton, R. O., and McEwen, J. E. (1996). Crosstalk between nuclear and mitochondrial genomes. Annu. Rev. Biochem. 65, 563-607.

Rothermel, B. A., Thornton, J. L., and Butow, R. A. (1997). Rtg3p, a basic helix-loop-helix/leucine zipper protein that functions in mitochondrial-induced changes in gene expression, contains independent activation domains. J. Biol. Chem. 272, 19801-19807.

Sekito, T., Thornton, J., and Butow, R. A. (2000). Mitochondria-to-nuclear signaling is regulated by the subcellular localization of the transcription factors Rtglp and Rtg3p. Mol. Biol. Cell 11, 2103-2115.

Sickmann, A., Reinders, J., Wagner, Y., Joppich, C., Zahedi, R., Meyer, H. E., Schonfisch, B., Perschil, I., Chacinska, A., Guiard, B., Rehling, P., Pfanner, N., and Meisinger, C. (2003). The proteome of Saccharomyces cerevisiae mitochondria. Proc. Natl. Acad. Sci. U.S.A. 100, 13207-13212.

Sinclair, D. A., and Guarente, L. (1997). Extrachromosomal rDNA circles a cause of aging in yeast. Cell 91 , 1033-1042.

Somsen, O. J. G., Hoeben, M. A., Esgalhado, E., Snoep, J. L., Visser, D., van der Heijden, R. T. J. M., Heijnen, J. J., and Westerhoff, H. V. (2000). Glucose and the ATP paradox in yeast. Biochem. J. 352, 593-599.

Sun, J., Kale, S. P., Childress, A. M. Pinswasdi, C., and Jazwinski, S. M. (1994). Divergent roles of RAS1 and RAS2 in yeast longevity. J. Biol. Chem. 269, 18638-18645.

Veatch, J. R., McMurray, M. A., Nelson, Z. W., and Gottschling, D. E. (2009). Mitochondrial dysfunction leads to nuclear genome instability via an iron-sulfur cluster defect. Cell 137, 1247-1258.

Wang, J., Jiang, J. C., and Jazwinski, S. M. (2010). Gene regulatory changes in yeast during life extension by nutrient limitation. Exp. Gerontol. 45, 621-631.

Woo, D. K., and Poyton, R. O. (2009). The absence of a mitochondrial genome in rho0 yeast cells extends lifespan independently of retrograde regulation. Exp. Gerontol. 44, 390-397.

Conflict of Interest Statement: The authors declare that the research was conducted in the absence of any commercial or financial relationships that could be construed as a potential conflict of interest.

Received: 21 October 2011; accepted: 17 December 2011; published online: 10 January 2012.

Citation: Miceli MV, Jiang JC, Tiwari A, Rodriguez-Quiñones JF and Jazwinski SM (2012) Loss of mitochondrial membrane potential triggers the retrograde response extending yeast replicative lifespan. Front. Gene. 2:102. doi: 10.3389/fgene. 2011.00102

This article was submitted to Frontiers in Genetics of Aging, a specialty of Frontiers in Genetics.

Copyright (C) 2012 Miceli, Jiang, Tiwari, Rodriguez-Quiñones and Jazwinski. This is an open-access article distributed under the terms of the Creative Commons Attribution Non Commercial License, which permits non-commercial use, distribution, and reproduction in other forums, provided the original authors and source are credited. 


\section{APPENDIX}

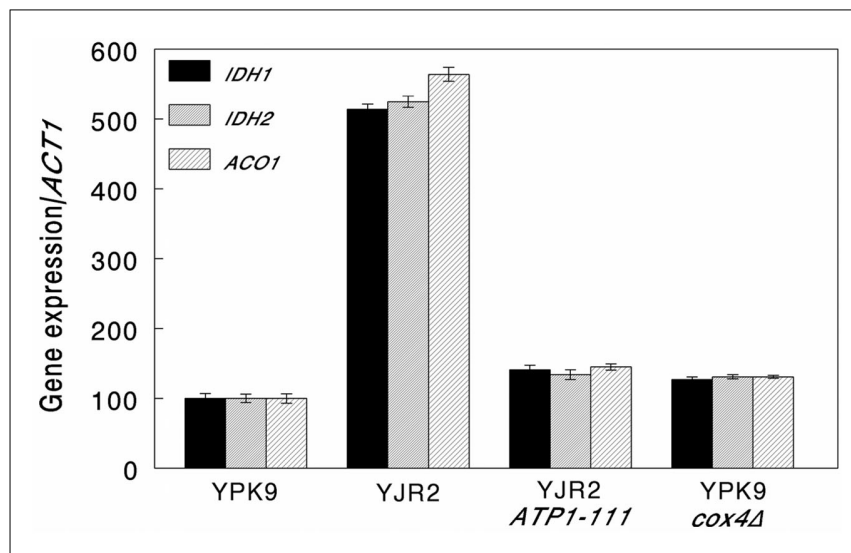

FIGURE A1 | Expression of retrograde response target genes.

Expression of $I D H 1, I D H 2$, and $A C O 1$ relative to ACT1 was determined by qRT-PCR as described in Section "Materials and Methods." The strains used were as follows: YPK9 $\left(\mathrm{rho}^{+}\right)$, YJR2 $\left(\mathrm{rho}^{\circ}\right)$, YJR2 ATP1-111, and YPK9 $\operatorname{cox} 4 \triangle$. Error bars are SEM for $N=6$ determinations. Expression of $I D H 1$, $I D H 2$, and $A C O 1$ increased in YJR2 compared to YPK9 $\left(P=3.96 \times 10^{-4}\right.$, $3.06 \times 10^{-4}$, and 0.0024 , respectively) and in YPK9 cox $4 \Delta$ compared to YPK9 ( $P=0.0102,0.0081$, and 0.0051 , respectively). Expression of $I D H 1$, $1 \mathrm{DH} 2$, and $A C O 1$ decreased in YJR2 ATP1-111 compared to YJR2 $\left(P=1.08 \times 10^{-4}, 2.73 \times 10^{-4}\right.$, and 0.0021 , respectively). $P$-values are adjusted for multiple comparisons. 